\title{
Rigorous development of computer based Integrated Care Pathways for HIV patients
}

\author{
Petros Papapanagiotou \\ University of Edinburgh \\ pe.p@ed.ac.uk
}

\author{
Jacques Fleuriot \\ University of Edinburgh \\ jdf@inf.ed.ac.uk
}

\author{
Areti Manataki \\ University of Edinburgh \\ A.Manataki@ed.ac.uk
}

\author{
Andrew Winter \\ NHS Greater Glasgow and Clyde \\ andrew.winter@nhs.net
}

NHS Greater Glasgow and Clyde rak.nandwani@nhs.net

\author{
Daniel Clutterbuck \\ NHS Lothian \\ daniel.clutterbuck@nhs.net
}

\author{
David Wilks \\ NHS Lothian \\ david.wilks@nhslothian.scot.nhs.uk
}

\begin{abstract}
We describe ongoing work on the modelling, verification, and implementation of Integrated Care Pathways (ICPs) for HIV patients in Scotland. Healthcare knowledge, including existing policies, guidelines, and best practices, is encoded in formal, process-based, workflow models. This is achieved through the use of our logic-based, modelling framework WorkflowFM, which provides high levels of rigour and trust about the designed ICPs. We believe this effort will result in a formally verified, easily maintainable, computer based implementation of HIV ICPs that will greatly improve the current standard of care.
\end{abstract}

integrated care pathways, human immunodeficiency virus, workflow, artificial intelligence

\section{INTRODUCTION}

HIV infection is of major public health importance, causing 1.6 million deaths worldwide in 2012 alone [3]. Integrated care pathways (ICPs) are thus becoming increasingly important for providing effective HIV care [2]. Such pathways are known to support the planning, organisation, and integration of HIV services, while enforcing clinical guidelines and hospital policies. They help automate documentation and guide healthcare practitioners through different routes of care, thus supporting the safety and continuity of care.

Despite the widely recognised importance of HIV ICPs, there is limited work in this area to demonstrate rigorous development of such pathways and there is virtually no formal evaluation of the best methodologies. The majority of existing studies provide a visual or textual representation the ICPs in a wide variety of form, scope, focus, and level of abstraction (see examples $[1,5]$ ).

In this work, we are using innovative technologies to model, simulate, and implement HIV ICPS. Our project brings together the knowledge and expertise of 2 types of specialists. On the one hand, experts in formally verified process modelling provide the means for rigorous workflow models for ICPs. On the other hand, HIV specialists with years of experience on everyday HIV patient care provide extensive knowledge of the involved medical procedures and care guidelines, and a clear picture of the existing standard of health, its pitfalls, and the necessary steps for improvement.

\section{HIV ICP DEVELOPMENT IN SCOTLAND}

In Scotland there is an ongoing effort to develop HIV ICPs for the first 3 months of care for all newly- diagnosed patients as part of the HIV Implementation and Improvement Programme.

In particular, recent efforts in NHS Greater Glasgow \& Clyde have resulted on a mapped, paper-based model of the involved processes. This model has been developed based on the experience of HIV clinicians and on patient involvement, and incorporates important care aspects that complement clinical assessments and decisionmaking, such as information governance and selfmanagement support.

In NHS Lothian, the effort towards developing HIV ICPs, has resulted in a paper-based implementation that has been supporting interdisciplinary HIV care since May 2013 [7].

Our work builds on top of these efforts to deliver a rigorous, computer-based model and implementation of HIV ICPS. We describe the underlying technology next.

\section{RIGOROUS WORKFLOW MODELS FOR ICPS}

In recent work, we presented a rigorous methodology for the development of formally verified, correct-by-construction workflows of healthcare processes [6]. Our approach, called WorkflowFM, has been applied to the modelling of patient handovers (assignment and delegation of healthcare services) [6] as well as intra-hospital transfers of tracheostomy patients [4]. Our system makes use of logic-based techniques to provide the following unique capabilities for each modelled ICP:

- A diagrammatic, process model that clearly depicts the involved steps in various levels of abstraction.

- Rigorous enforcement of policies \& guidelines. 
- Mathematical guarantees of consistency and continuity with respect to the original conceptualisation.

- Systematic accounting of resources, information flow tracking, and explicit recording of exceptions and variances.

- Simulated execution of various scenarios.

- Automated deployment as a central coordinator that can integrate with existing infrastructure and mobile devices.

We believe WorkflowFM with its associated artificial intelligence engine and modelling methodology can provide high levels of trust and assurances about the developed ICPS.

\section{GOAL AND METHODOLOGY}

The goal of our project is to model, simulate, and implement ICPs for HIV patients in Scotland with the use of WorkflowFM, in order to allow the provision of patient-centred, safe, continuous, efficient care. More specifically, we focus on a process-based analysis of the first 3 months of HIV care, including the identification of variances, in order to construct a model that systematically elicits policies, guidelines, and best practices. This can then be deployed as a software system that supports HIV practitioners and contributes to a high standard of care. In our efforts to accomplish these goals, we follow a standard, 4stage methodology.

1) First, we consult with all the stakeholders involved in HIV care, including clinicians, nurses, and administrators, to breakdown and formalise the care processes. We draw information from existing studies, contextual interviews and shadowing.

2) We then model the mapped processes in WorkflowFM, and compose them into workflows.

3) Next, we run sample simulations in order to empirically verify the correctness of the workflow.

4) Lastly, we deploy the ICP models as a centrally coordinated, integrated, electronic, mobile platform.

Based on our previous experience, each stage has its own set of challenges. We believe the combined expertise of the members of our project is key for tackling these challenges effectively and improving the standard of care for HIV patients in Scotland.

\section{CURRENT PROGRESS}

We are currently performing the first and second stages of the 4-stage methodology described above. Regular meetings of the current multi-disciplinary team have resulted in an initial, evolving model of the first 3 months of HIV patient care. This currently contains 3 layers of abstraction, where each process at a high level is broken down to a set of connected processes at the next level.

The visual workflow representation helps the clinical members of our team identify implicit dependencies and reflect upon their practices. In addition, we discuss variances that may occur at any stage of the ICP (e.g. inconclusive tests and patient no-shows) and how they should be handled.

\section{CONCLUSION}

The significance of ICPs in poviding improved, effective care for HIV patients has been recognised by a number of NHS boards and organisations. We combine state-of-the-art technologies for rigorous workflow modelling with the expertise of HIV specialists in Scotland to develop computer based HIV ICPs for the first 3 months of care. Our logicbased WorkflowFM tool used throughout the stages of this project provides high levels of assurances about various properties of the modelled ICPs. Our ongoing project is showing promising results towards an improved, safe, effective, continuous provision of care for HIV patients in Scotland.

\section{ACKNOWLEDGMENTS}

This research was supported by EPSRC grant EP/J001058/1 and the Centre for Intelligent Systems and their Applications, University of Edinburgh. We also thank Dr. Alexandrea Macpherson of NHSGG\&C for her input.

\section{REFERENCES}

[1] Griffiths C., Miles K., Aldam D., Cornforth D., Minton J., Edwards S., Williams I. (2007) A nurse- and pharmacist-led treatment advice clinic for patients attending an HIV outpatient clinic. Journal of advanced nursing, 58(4), 320-326.

[2] Healthcare Improvement Scotland. (2011) Standards for Human Immunodeficiency Virus (HIV) services. http://www.healthcareimprove mentscotland.org/our_work/long_term_condition s/hiv_treatment_and_care/hiv_standards.aspx

[3] Joint United Nations Programme on HIV/AIDS (UNAIDS). (2013) Global report: UNAIDS report on the global AIDS epidemic. http://www.unaids .org/en/resources/documents/2013/20130923_U NAIDS_Global_Report_2013

[4] Manataki A., Fleuriot J., Papapanagiotou P. (2014) Tracheostomy transfers: A case study in the application of formal methods to intra-hospital patient transfers. 27th International Symposium on Computer-Based Medical Systems (CBMS), New York, 257-262. IEEE.

[5] NICE pathways. (2014) HIV testing and prevention overview. http://pathways.nice.org.uk/ pathways/hiv-testing-and-prevention

[6] Papapanagiotou P., Fleuriot J.D. (2013) Formal verification of collaboration patterns in healthcare. Behaviour \& Information Technology.

[7] Wielding S., Clutterbuck D.J., Wilks D., Panton L. (2013) Development of an ICP for HIV Outpatient care in Lothian, Scotland. European Care Pathways Conference, Glasgow. 